\title{
GLORIFIED OPTICS AND WAVE PROPAGATION IN NONPLANAR STRUCTURE
}

\author{
By TaI-Lin Hong and Donald V. Helmberger
}

\begin{abstract}
Waves propagating in varying nonplanar structure can produce many interesting phenomena, such as focusing, caustics, and triplications. A high-frequency technique based on the first-motion approximation, referred to as glorified optics, has been developed to generate synthetic seismograms for these types of problems. The technique, in its simplest form, uses the spreading rate of a beam with transmission and reflection coefficients along each possible ray path. The time behavior of each arrival is either that of the original pulse or its Hilbert transform depending on the position of caustics. The geophysically interesting structure of a soft basin over a half-space is investigated in detail by this method. Synthetic seismograms appropriate for various locations are compared with the results of finite difference and finite element methods. The technique appears rich in insight and should prove very useful in modeling problems.
\end{abstract}

\section{INTRODUCTION}

In recent years there has been a substantial increase in the number of seismic wave-form inversion studies. These efforts are characterized by matching a set of records by computing synthetic wave forms for the forward problem and matching the observations by a trial and error procedure. The fits for some of the more diligent efforts are sufficiently good to allow the application of linearized inversion techniques (see for example Burdick and Mellman, 1976). Unfortunately, in many studies the matching procedure can be frustrating due to the presence of scattered waves which can be readily detected by particle motion plots. However, one does not need to do much soul searching to discover abundant causes, such as basins and ridges above the source and/or similar receiver structure. The effects of basins, for example, have been well studied by Aki and Larner (1970) and Boore et al. (1971). But, because these numerical methods are so expensive one can hardly perform a parameter search to use in wave-form inversion studies especially when these wave forms contain high frequency. Furthermore, most numerical methods do little to develop ones insight with respect to the physics of the problem. Thus, we have developed a technique of generating synthetics by simply tracking rays. The method is based on first-motion approximations and is, therefore, a high-frequency solution but appears to compare favorably to numerical experiments even at long periods in many situations.

\section{THEORY}

Solving rigorously the boundary value problem involving complicated geometry is not possible, so we have jumped directly to an approximate form of the solution. The approximation is based on the understanding of the connection between the physical behavior of rays and the mathematical formulation for the solution of simple structures. We will introduce our technique by first reviewing the interface problem, followed by multi-dipping layers, and then generalize to smoothly varying interfaces. 
Fluid interface problem. We assume a point source with time history given by $f(t)$ with the geometry specified in Figure 1. Applying generalized ray theory we can write down the high-frequency solution, Helmberger (1968)

$$
\phi(r, z, t)=f(t) * \sqrt{\frac{2}{r}} \frac{1}{\pi} \frac{d}{d t}\left[\frac{1}{\sqrt{t}} * \operatorname{Im}\left(T(p) \frac{\sqrt{p}}{\eta_{1}} \frac{d p}{d t}\right)_{\Gamma}\right]
$$

where $\phi$ is a scalar potential

$$
\begin{aligned}
T(p) & =\text { transmission coefficient } \\
p & =\text { complex ray parameter } \\
\Gamma & =\text { de-Hoop contour } \\
\eta_{i} & =\left(\frac{1}{v_{i}^{2}}-p^{2}\right)^{1 / 2}
\end{aligned}
$$

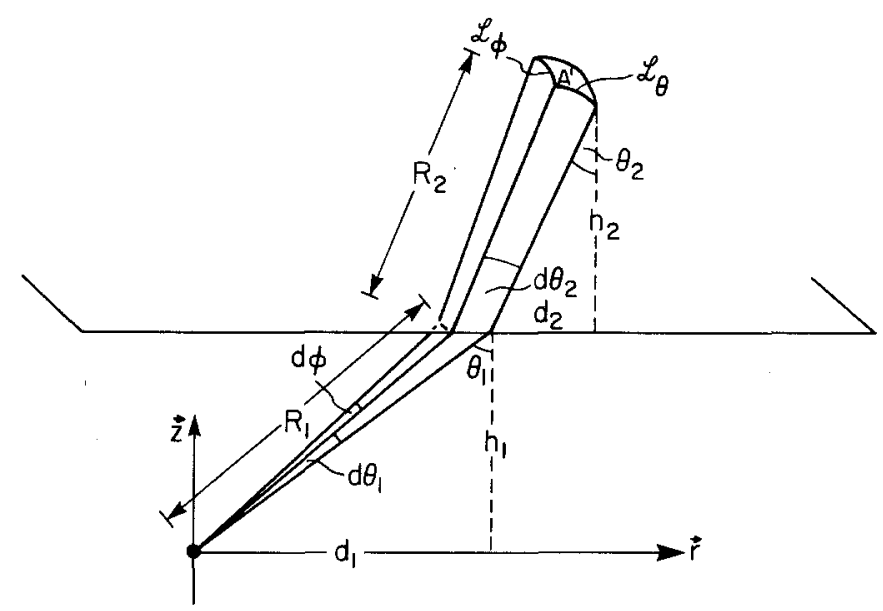

FIG. 1. Diagram displaying the infinitesimal phenomena of geometric spreading for the simple interface problem. The area $A^{\prime}$ is the product of $\mathscr{L}_{\theta}$ and $\mathscr{L}_{\phi}$ where $\mathscr{L}_{\theta}=R_{1} d \theta_{1}\left(\cos \theta_{2} / \cos \theta_{1}\right)+R_{2} d \theta_{2}$ and $\mathscr{L}_{\phi}=R_{1} d \phi \sin \theta_{1}+R_{2} d \phi \sin \theta_{2}$.

This solution can be simplified further by constructing the first-motion approximation for times near the direct arrival, $t_{0}\left(p_{0}\right)$

$$
\frac{d p}{d t} \approx \frac{i}{\left(2\left(t-t_{0}\right)\right)^{1 / 2}}\left(\frac{d^{2} t}{d p^{2}}\right)_{t=t_{0}}^{-1 / 2}
$$

But from the geometry given in Figure 1 we can show that the spreading factor, $S_{f}$

$$
S_{f} \equiv\left\{\sqrt{\frac{p}{r}} \frac{1}{\eta_{1}}\left[\left|\frac{d^{2} t}{d p^{2}}\right|\right]^{-1 / 2}\right\}_{t=t_{0}}=\left(\frac{A}{A_{0}}\right)^{-1 / 2}
$$

where $A_{0}$ is the original cross-sectional area of the beam of rays at unit distance and 
$A$ is the projected area at the receiver. To show this we note that

$$
\begin{gathered}
\eta_{1}\left(\left|\frac{d^{2} t}{d p^{2}}\right|\right)^{1 / 2}=\eta_{1}\left(\frac{h_{1}}{\left.{\eta_{1}{ }^{3} v_{1}{ }^{2}}^{1 / 2}+\frac{h_{2}}{\eta_{2}{ }^{3} v_{2}{ }^{2}}\right)^{1 / 2}}\right. \\
{\left[\eta_{1}\left(\left|\frac{d^{2} t}{d p^{2}}\right|\right)^{1 / 2}\right]_{t=t_{0}}=\frac{1}{\sqrt{v_{1}}}\left[R_{1} d \theta_{1}+R_{2} d \theta_{2} \frac{\cos \theta_{1}}{\cos \theta_{2}}\right]^{1 / 2} \cdot \frac{1}{\sqrt{d \theta_{1}}}}
\end{gathered}
$$

and

$$
\left(\sqrt{\frac{\bar{r}}{p}}\right)_{t=t_{0}}=\left[\frac{R_{1} \sin \theta_{1}+R_{2} \sin \theta_{2}}{\sin \theta_{1}}\right]^{1 / 2} \sqrt{\mathrm{v}_{1}}
$$

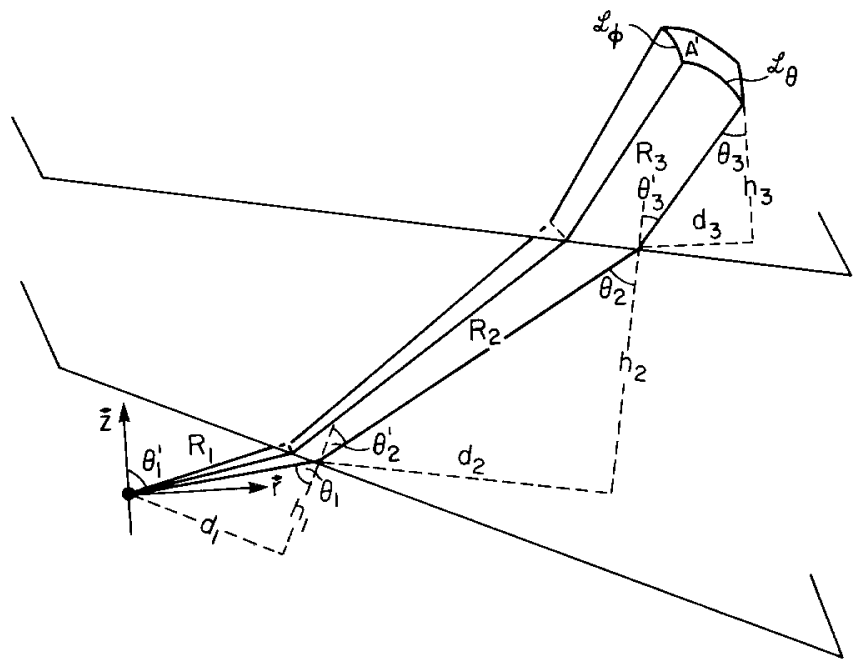

FIG. 2. Diagram displaying the infinitesimal phenomena of geometric spreading for dipping interfaces. The area $A^{\prime}$ is the product of $\mathscr{L}_{\theta}$ and $\mathscr{L}_{\phi}$ where

$$
\begin{aligned}
\mathscr{L}_{\theta}= & R_{1} d \theta_{1}\left[\left(\cos \theta_{2}^{\prime} \cos \theta_{3}\right) /\left(\cos \theta_{1} \cos \theta_{2}\right)\right] \\
& +R_{2} d \theta_{2}\left(\cos \theta_{3}^{\prime} / \cos \theta_{2}\right)+R_{3} d \theta_{3} \\
\mathscr{L}_{\phi}= & R_{1} \sin \theta_{1} d \phi+R_{2} \sin \theta_{2} \frac{\sin \theta_{2}^{\prime}}{\sin \theta_{2}} d \phi+R_{3} \sin \theta_{3} \frac{\sin \theta_{3}}{\sin \theta_{3}} \cdot \frac{\sin \theta_{2}^{\prime}}{\sin \theta_{2}} d \phi
\end{aligned}
$$

Thus

$$
S_{f}=\left[\frac{\left(R_{1} \sin \theta_{1} d \phi+R_{2} \sin \theta_{2} d \phi\right)\left(R_{1} d \theta_{1}+R_{2} d \theta_{2} \cos \theta_{1} / \cos \theta_{2}\right)}{\sin \theta_{1} d \theta_{1} d \phi}\right]^{-1 / 2}
$$

but if we let $A=A^{\prime} \cos \theta_{1} / \cos \theta_{2}$ or the projection of $A^{\prime}$ onto the original orientation we obtain

$$
S_{f}=\left[\frac{A}{A_{0}}\right]^{-1 / 2}
$$


The solution becomes

$$
\phi=f(t) * \frac{1}{\pi} \frac{d}{d t}\left[\frac{1}{\sqrt{t}} * \frac{1}{\sqrt{t-t_{0}}}\right] \mathscr{R} e\left(T\left(p_{0}\right)\right) \cdot S_{f}
$$

which reduced to

$$
\phi=f\left(t-t_{0}\right)\left(\frac{A}{A_{0}}\right)^{-1 / 2} \mathscr{R} e\left[T\left(p_{0}\right)\right]
$$

Note that the velocity contrast across the interface changes $d \theta_{1}$ to $d \theta_{2}$ or the spreading rate in the $\theta$-direction, whereas $d \phi$ remains the same.

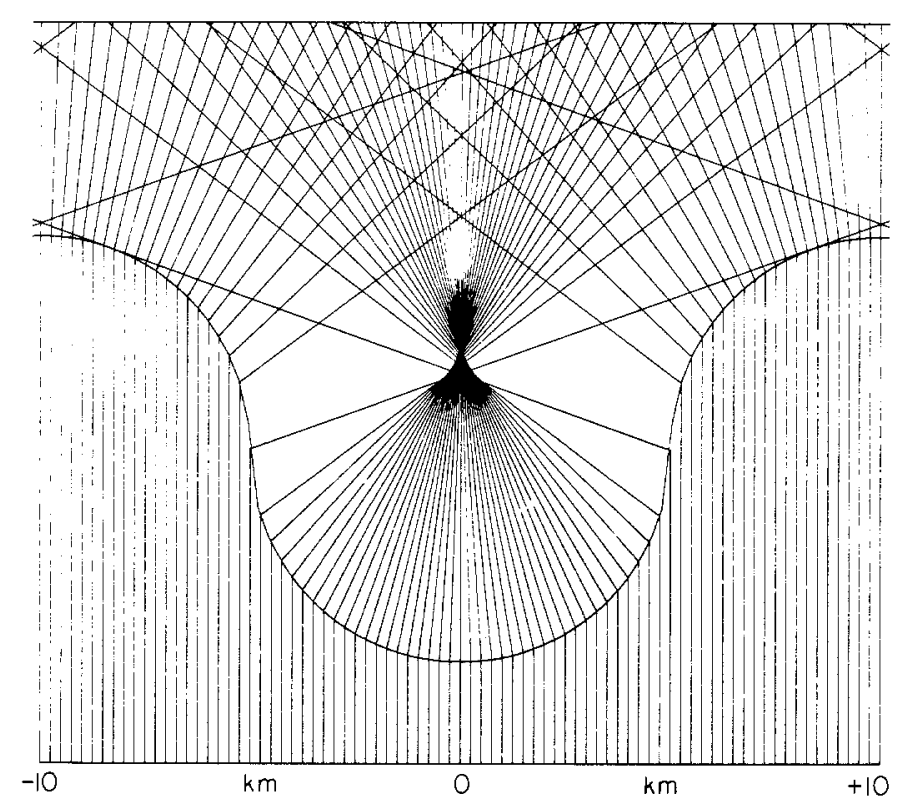

FIG. 3. Diagram showing the bending of geometric ray paths caused by a periodic semicircular interface.

Dipping structure. We now consider a more general case, namely two nonparallel planar interfaces. This problem was studied earlier by Hong and Helmberger (1977) assuming a line source excitation. For a point source, we require an additional complication due to spreading in the azimuthal direction. For flat structure this correction is simply $\sqrt{p / r}$ but for dipping structure we must represent it in terms of parameters in local Cartesian coordinates

$$
\phi=f(t) * \frac{\sqrt{2}}{\pi} \frac{d}{d t}\left[\frac{1}{\sqrt{t}} * \operatorname{Im}\left(T(p) \cdot\left(\sum_{m=1}^{3} \frac{d_{m}}{p_{m}}\right)^{-1 / 2} \frac{1}{\eta_{1}} \frac{d p_{1}}{d t}\right)_{\Gamma}\right]
$$

where $p_{m}$ is the local ray parameter of the $m$-th ray segment and $d_{m}$ is the projection of the geometric path onto the local Cartesian coordinates, as shown in Figure 2. 
The justification for this factor along with the details of the contour $\Gamma$ are given in our earlier paper. Again, we take the first motion approximation by letting

$$
\frac{d p_{1}}{d t} \approx \frac{i}{\left(2\left(t-t_{0}\right)\right)^{1 / 2}}\left(\left|\frac{d^{2} t}{d p_{1}^{2}}\right|\right)^{-1 / 2}
$$

and after some algebra (see Appendix) we obtain

$$
\eta_{1}\left|\frac{d^{2} t}{d p_{1}^{2}}\right|^{1 / 2}=\frac{1}{\sqrt{v_{1}}}\left[R_{1} d \theta_{1}+R_{2} d \theta_{2} \frac{\cos \theta_{1}}{\cos \theta_{2}^{\prime}}+R_{3} d \theta_{3} \frac{\cos \theta_{2}}{\cos \theta_{3}^{\prime}} \frac{\cos \theta_{1}}{\cos \theta_{2}^{\prime}}\right]^{1 / 2} \frac{1}{\sqrt{d \theta_{1}}}
$$

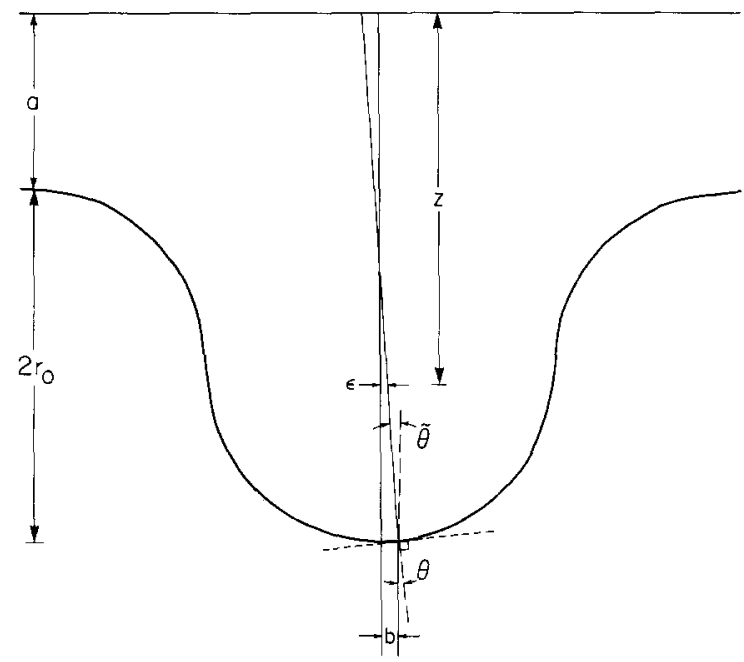

FIG. 4. Diagram displaying the infinitesimal phenomena of geometric spreading for a curved boundary. The parameters are $a=5 \mathrm{~km}, r_{0}=5 \mathrm{~km}$, the upper velocity $\left(v_{1}\right)$ is $1 \mathrm{~km} / \mathrm{sec}$ and the lower velocity $\left(v_{2}\right)$ is $3 \mathrm{~km} / \mathrm{sec}$, and the density is assumed constant.

and

$$
\left(\sum_{m=1}^{3} \frac{d_{m}}{p_{m}}\right)^{1 / 2}=\frac{\left(d_{1}+d_{2} \frac{\sin \theta_{2}^{\prime}}{\sin \theta_{2}}+d_{3} \frac{\sin \theta_{3}^{\prime}}{\sin \theta_{3}} \frac{\sin \theta_{2}^{\prime}}{\sin \theta_{2}}\right)^{1 / 2} \cdot\left(v_{1}\right)^{1 / 2}}{\left(\sin \theta_{1}\right)^{1 / 2}}
$$

thus

$$
S_{f} \equiv\left(\sum_{m=1}^{3} \frac{d_{m}}{p_{m}}\right)^{-1 / 2} \frac{1}{\eta_{1}}\left(\left|\frac{d^{2} t}{d p_{1}^{2}}\right|\right)^{-1 / 2}=\left(\frac{A}{A_{0}}\right)^{-1 / 2}
$$

where

$$
A=A^{\prime} \frac{\cos \theta_{1}}{\cos \theta_{2}^{\prime}} \frac{\cos \theta_{2}}{\cos \theta_{3}^{\prime}}
$$


or the projection of $A^{\prime}$ given in Figure 2 back into the original orientation. The solution becomes

$$
\phi=f\left(t-t_{0}\right)\left(\frac{A}{A_{0}}\right)^{-1 / 2} \mathscr{R} e\left[T\left(p_{0}\right)\right]
$$

where we must compute the transmission coefficients with the appropriate local ray parameter $\left(p_{m}\right)$. Calculating $\left(A / A_{0}\right)$ analytically for a large number of ray segments

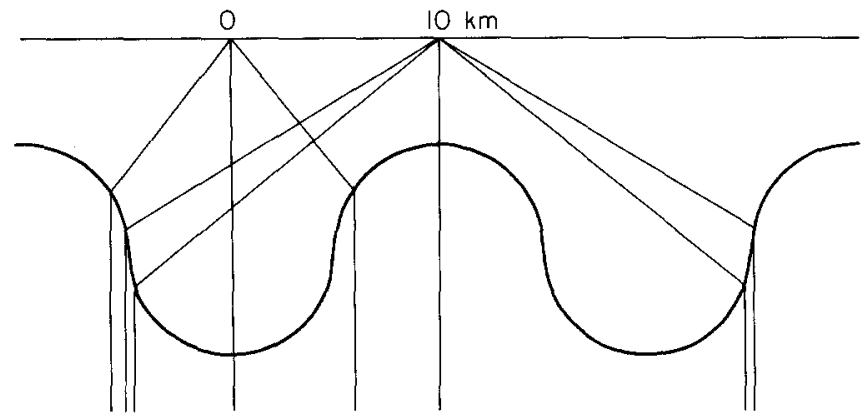

FIG. 5. Diagram showing the paths of geometric rays arriving at $x=0 \mathrm{~km}, z=0 \mathrm{~km}$, and $x=10 \mathrm{~km}$, $z=0 \mathrm{~km}$.

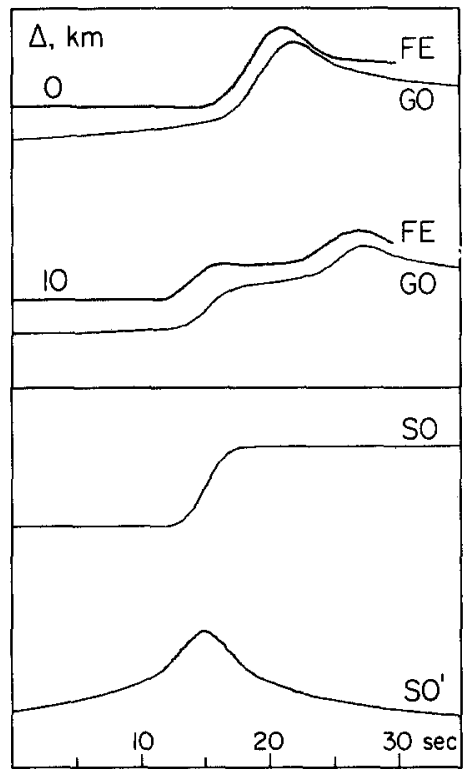

FIG. 6. Comparison of $S H$ displacements received at the two locations shown in Figure 5. The traces marked with FE are by finite element method. Those marked with GO are by glorified optics.

is laborious but could be done. A much more practical approach and one we recommend is to simply add a small $(\delta p)$ to the proper ray parameter, $p_{0}$, that $p$ required to track a ray from the source to the receiver and measure $\left(A^{\prime} / A_{0}\right)$ numerically, or more precisely

$$
\lim _{\delta p \rightarrow 0}\left(\frac{A}{A_{0}}\right)=\lim _{\delta p \rightarrow 0}\left(\frac{\mathscr{L}_{\theta}}{A_{0}}\right) \cdot \mathscr{L}_{\phi}\left[\frac{\cos \theta_{1}}{\cos \theta_{2}^{\prime}} \cdots \frac{\cos \theta_{n}}{\cos \theta_{n+1}^{\prime}}\right]
$$


where the azimuthal spreading

$$
\lim _{\delta \phi \rightarrow 0}\left\{\frac{\mathscr{L} \phi}{\delta \phi}\right\}=p_{1}\left(\sum_{m}\left(\frac{d_{m}}{p_{m}}\right)\right)
$$

can be computed analytically. The numerical procedure is required to obtain the limiting value on $\mathscr{L}_{\theta}$ when its behavior becomes highly complicated with the addition of curvature as we will demonstrate shortly.

Periodical semicircular boundary. In this section we apply our technique to a simple curved boundary such that the limiting process can be performed analytically in the presence of caustics. For convenience, we will assume a plane wave source at vertical incidence to eliminate $\mathscr{L}_{\phi}$ contributions. The geometrical considerations displaying focusing and other complications are given in Figure 3 . We begin as
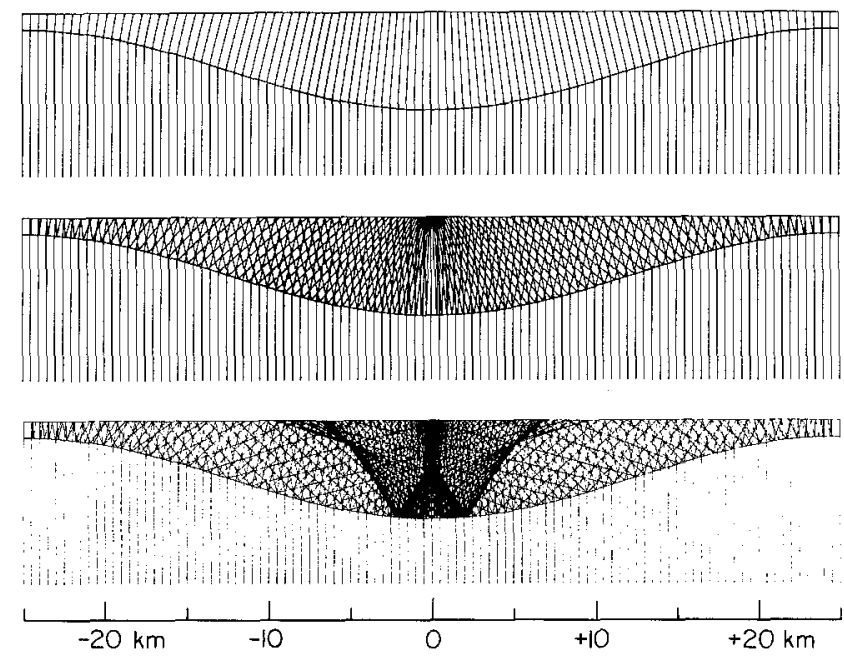

Fig. 7. Diagram showing the paths of geometric rays; the direct rays on the top; the rays with two reflections in the middle; the rays with four reflections on the bottom.

before by investigating the spreading rate for a particular ray as shown in Figure 4. The functional description of the boundary for the assumed coordinate system is

$$
\left[z-\left(a+r_{0}\right)\right]^{2}+x^{2}=r_{0}^{2}, \quad \text { for }-r_{0} \leqq x \leqq r .
$$

The angle between the local normal and vertical is

$$
\theta(x)=\tan ^{-1}\left(x /\left(r_{0}^{2}-x^{2}\right)^{1 / 2}\right)
$$

and, further, let

$$
\tilde{\theta}(x)=\theta-\sin ^{-1}\left(v_{1} \sin \theta / v_{2}\right)
$$

where $v_{1}$ and $v_{2}$ are the velocities in the upper and lower media, respectively. The spreading element, $\epsilon$, is

$$
\epsilon=b-\left[\left(a+r_{0}\right)+\left(r_{0}^{2}-b^{2}\right)^{1 / 2}-z\right] \tan \tilde{\theta}
$$


from the geometry given in Figure 4 . Next, we perform the limiting process

$$
\lim _{b \rightarrow 0} \frac{\epsilon}{b}=1-\left[a+2 r_{0}-z\right] \lim _{b \rightarrow 0}\left[\frac{d}{d b} \tan \tilde{\theta}\right]
$$

where

$$
\frac{d}{d b} \tan \tilde{\theta}=\frac{1}{\cos ^{2} \tilde{\theta}}\left[1-\frac{\frac{v_{1}}{v_{2}} \cos \theta}{\left(1-\frac{v_{1}^{2} \sin ^{2} \theta}{v_{2}^{2}}\right)^{1 / 2}}\right]\left(r_{0}^{2}-b^{2}\right)^{-1 / 2}
$$
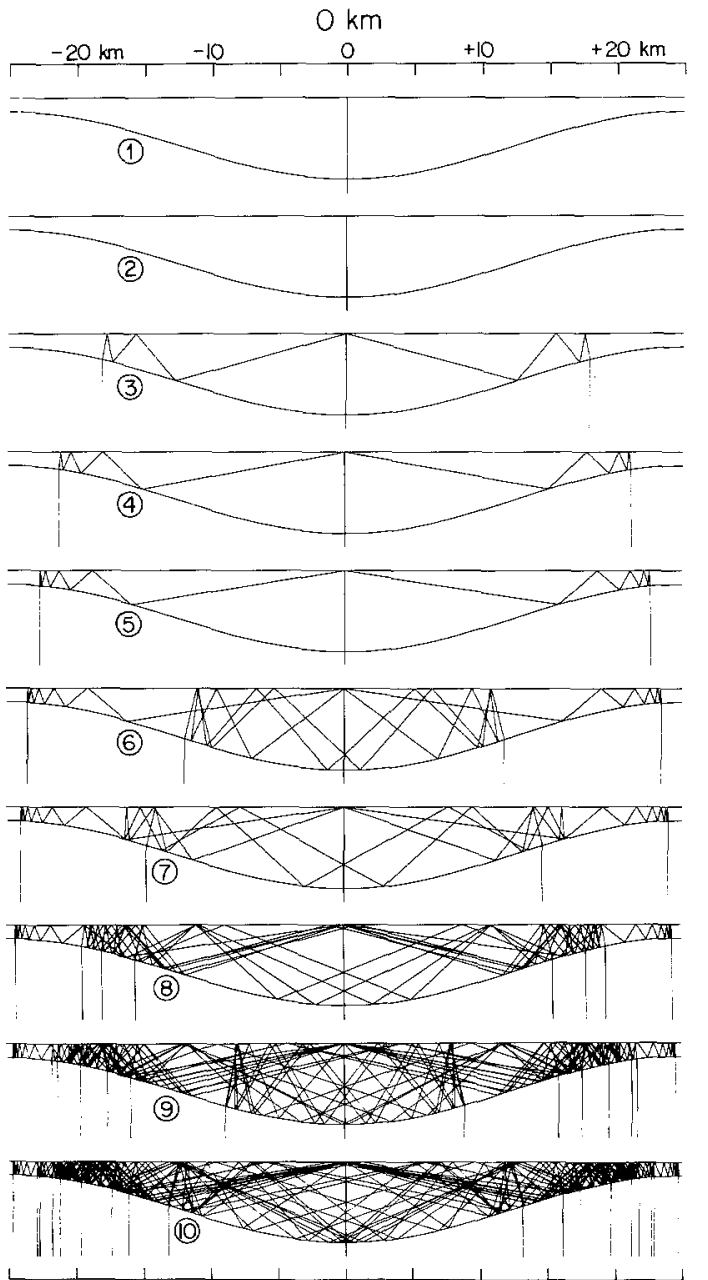

FIG. 8. The paths of geometric rays arriving at $x=0$. The top trace, (1), contains the direct ray, and (2) displays the ray with two reflections, etc.

Therefore

$$
S_{f}=\lim _{b \rightarrow 0}\left(\frac{A}{A_{0}}\right)^{-1 / 2}=\left[1-\left(a+2 r_{0}-z\right)\left(1-\frac{v_{1}}{v_{2}}\right) \frac{1}{r_{0}}\right]^{-1 / 2} .
$$


GLORIFIED OPTICS AND WAVE PROPAGATION IN NONPLANAR STRUCTURE 1321

Note that $A$ can be negative and there is a singular point along this geometric ray path with $z$ defined by

$$
z=a+2 r_{0}-r_{0} /\left(1-v_{1} / v_{2}\right)
$$

which is dramatically displayed in Figure 3. Our solution fails at this point but could be salvaged by using the third order saddle-point approximation. For

$$
z<a+2 r_{0}-r_{0} /\left(1-v_{1} / v_{2}\right)
$$

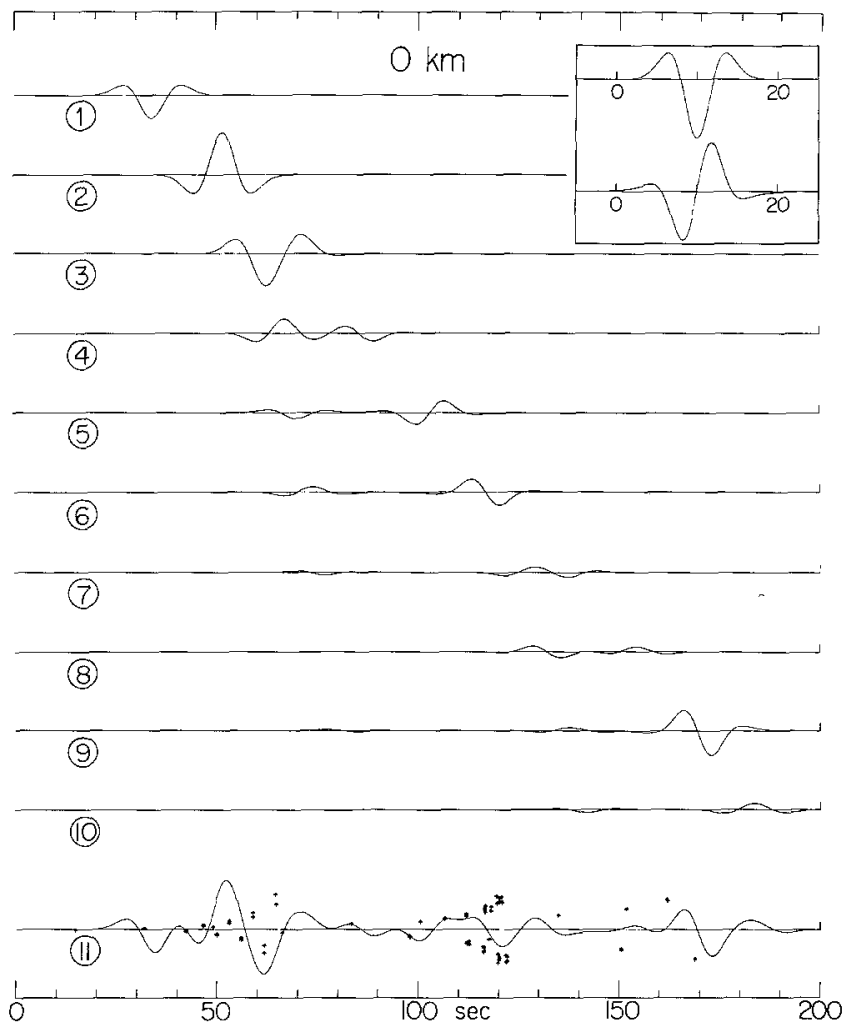

FIG. 9. Diagram showing the contribution of each set of rays displayed in Figure 8 with the final summation at the bottom. Arrows indicate arrival times.

we cross the caustic and for high frequencies the response can be approximated by a $90^{\circ}$ phase shift [see Hill (1974) for a discussion of such effects]. If the receiver is right on the caustic, the approximation fails and the tedious third-order approximation must be used. Physically, for an input pulse of finite duration, the response on the caustic should not be dramatically different from the response at nearly locations (see Chapman, 1976). Thus, the easiest remedy is to move the receiver slightly and avoid the problem.

In cases involving multiple reflections the generalized coefficient containing the product of all interactions expressed simply as $T(p)$ can become complex, so that in general for $\left(A / A_{0}\right)>0$

$$
\phi=f\left(t-t_{0}\right) \mathscr{R} e\left(T\left(p_{0}\right)\right)\left|S_{f}\right|+f^{*}\left(t-t_{0}\right) \operatorname{Im}\left(T\left(p_{0}\right)\right)\left|S_{f}\right| .
$$



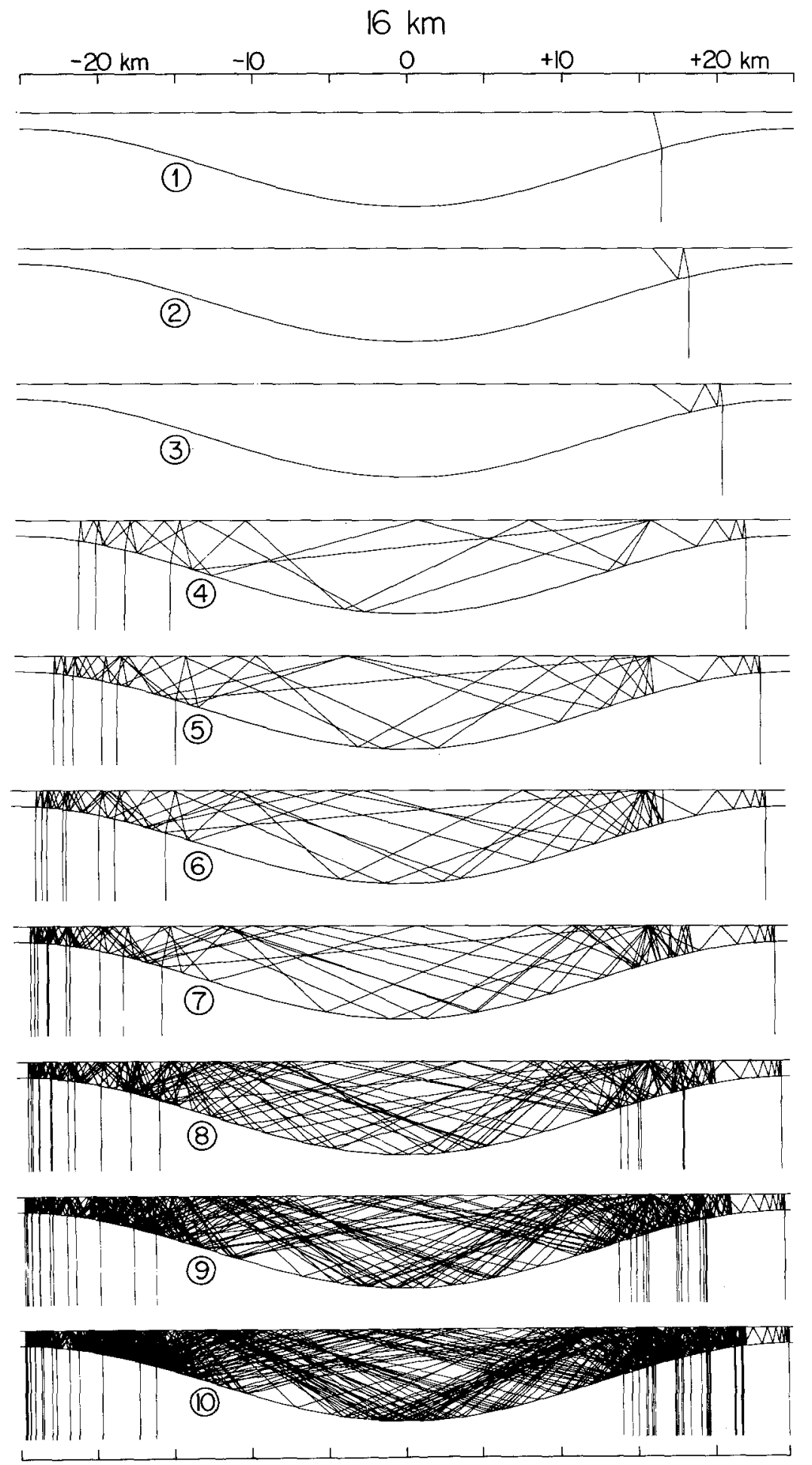

FIg. 10. The paths of geometric rays arriving at $x=16 \mathrm{~km}$. The top trace, (1), contains the direct ray and (2) displays the ray with two reflections, etc. 
Similarly, for $\left(A / A_{0}\right)<0$, the response becomes

$$
\phi=f^{*}\left(t-t_{0}\right) \mathscr{R} e\left(T\left(p_{0}\right)\right)\left|S_{f}\right|+f\left(t-t_{0}\right) \operatorname{Im}\left(T\left(p_{0}\right)\right)\left|S_{f}\right|
$$

where $f^{*}\left(t-t_{0}\right)$ is the allied function of $f\left(t-t_{0}\right)$, that is, the same amplitude spectra but with a $90^{\circ}$ phase shift.

In the next section we will generate synthetics for a soft basin over a half-space using several methods. Thus, as a preliminary comparison we compute the responses for the periodic structure at $x=0$ and $x=10 \mathrm{~km}$ as displayed in Figure 5. These two ranges were chosen to further test the phase shifts discussed above. Determining

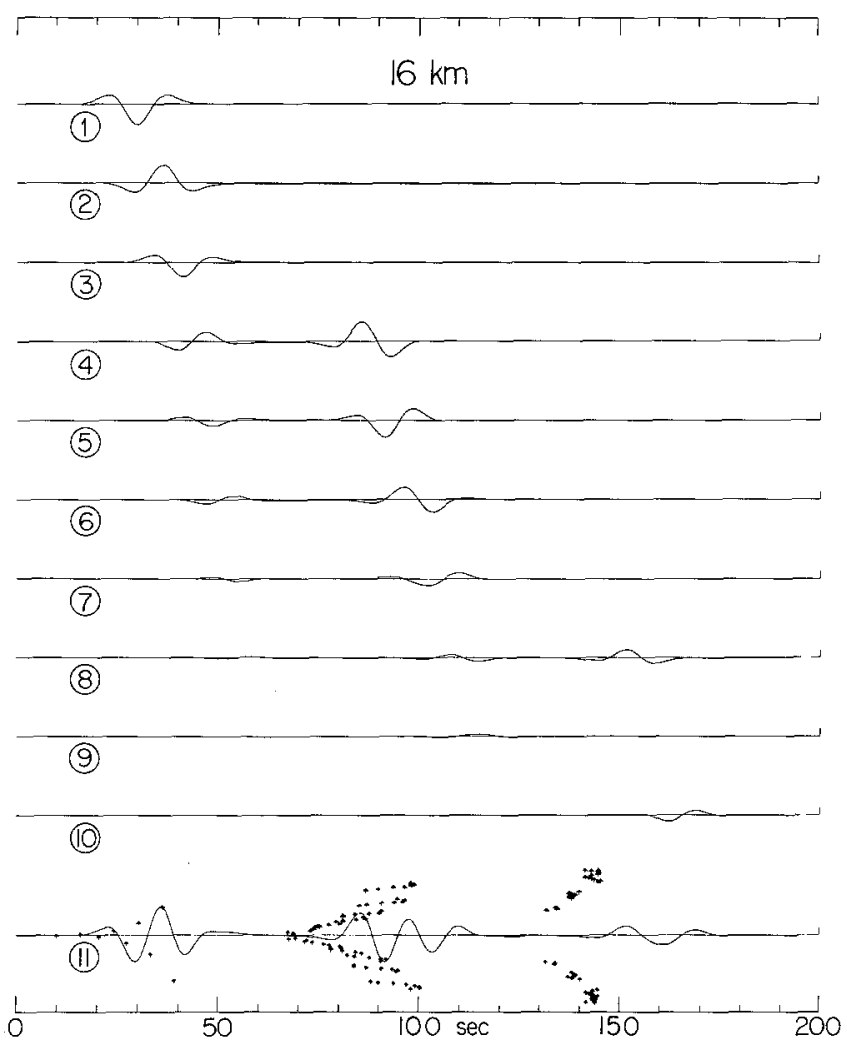

FIG. 11. Diagram showing the contribution of each set of rays displayed in Figure 10 with the final summation at the bottom. Arrows indicate arrival times.

the ray paths that lead to a particular location is achieved by a two-stage procedure. First, we set up a baseline from which we illuminate the area of interest such as Figure 3. Then we pick the rays nearest to an observation point and perform a fine tuning iteration scheme to find the ray parameter with the required accuracy. If we number the rays left to right we see that the second or middle ray is phase-shifted for $x=0$, whereas the second and fourth rays are phase-shifted at $x=10 \mathrm{~km}$. Glorified optics results (GO) are obtained by simply adding the rays shown in Figure 5 applying equations (23) and (24). The source function and its allied function are displayed at the bottom of Figure 6 with the synthetics given above along with the finite element results. The comparison is good considering the nature of the source. 
That is, $\mathrm{GO}$ are expected to give the most accurate result for short periods, thus the drift effects occurring in the GO synthetics are caused by the large offset in the assumed source. There are, also, errors associated with using the flat-layer transmission coefficient which could be easily corrected for local curvature. However, we are primarily concerned with focusing and defocusing in this particular study since such effects appear dominant.

\section{Numerical Results for a Basin}

Since our technique is a first-order approximation it is prudent to test its validity against other more exact techniques such as the numerical methods discussed by

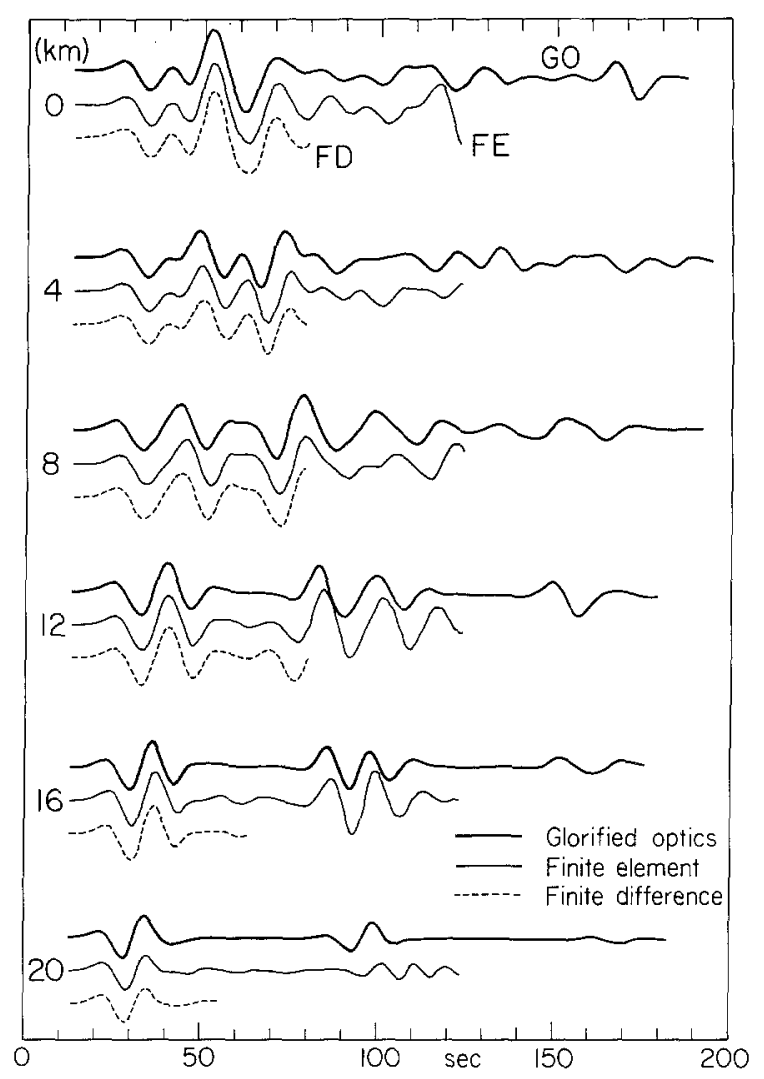

FIG. 12. The comparison between the results of glorified optics, finite element, and finite difference. The later results were obtained from Larner (1970, Figure 4.9). The traces are the tangential displacement at surface receivers with horizontal distances of 0 to $20 \mathrm{~km}$ from the center of the basin.

Aki and Larner (1970), the finite difference method, Boore et al. (1971) or the finite element method, Hong and Kosloff (1978). These numerical methods are basically long-period techniques in that the grid size must be large enough to make the method affordable. On the other hand, glorified optics become increasingly accurate as the frequency is increased, thus the various methods complement each other. Boore et al. (1971) have already performed a detailed comparison between the AL method and finite difference results for basin structures. We will attempt to match Boore's results using their model parameters and source description specified below. 
The curved boundary describing the bottom of the basin is

$$
\begin{array}{ll}
z(x)=D+\frac{C}{2}\left[1-\cos \left(2 \pi\left(x-\frac{w}{2}\right) / w\right)\right], & \text { for }-\frac{w}{2} \leqq x \leqq \frac{w}{2} \\
z(x)=D, & \text { elsewhere }
\end{array}
$$

where $w=50 \mathrm{~km}, D=1 \mathrm{~km}$, and $C=5 \mathrm{~km}$. A plane $S H$ wave with source time function, $f(t)$, is impinging vertically from the half-space, where

$$
f(t)=\frac{\sqrt{\pi}}{2}\left(a-\frac{1}{2}\right) \exp (-a)
$$

and

$$
\begin{aligned}
& a=6\left(t-t_{s}\right)^{2} /\left(T_{p} \cdot \frac{\sqrt{6}}{\pi}\right)^{2} \\
& t_{s}=20 \mathrm{sec}, \quad T_{p}=18.3 \mathrm{sec} .
\end{aligned}
$$

The density and velocity in the basin are $\rho_{1}=2 \mathrm{gm} / \mathrm{cm}^{3}, \beta_{1}=0.7 \mathrm{~km} / \mathrm{sec}$, respectively, and $\rho_{2}=3.3 \mathrm{gm} / \mathrm{cm}^{3}, \beta_{2}=3.5 \mathrm{~km} / \mathrm{sec}$ in the half-space.

The basin structure with the direct, and two sets of multiply reflected ray paths are displayed in Figure 7. The qualitative characteristics of the motion can be seen by observing the focusing of the multiples and development of caustics. For instance, the first multiples at the center of the basin focus rather strongly which will give rise to a large second arrival at this position. For longer times, rays begin to come in from the sides. Since our model is a ray method it is educational to observe the development of the motion as a function of ray summation for a couple of positions (see Figures 8 and 9 for the center position and Figures 10 and 11 for a station close to the edge of the basin). In Figure 8 , we display the rays by the number of times they are reflected internally starting at the top; that is (1) contains the direct, (2) contains one multiple and one ray, (3) contains two multiples but three rays, etc. The synthetic contribution from (1), (2), (3), etc. are displayed in Figure 9 individually with the final summation of all responses given at the bottom. Note that the rays bouncing vertically contribute very little after two reflections whereas the rays coming in from the sides are heavy contributors at large times because of the large low-angle reflections. The rays that enter on one side of the basin and travel across the basin internally and return again are sometimes large but usually small depending on focusing. The observations at the edge, or off center, are more interesting in many ways due to the large contributions that come from the opposite side of the basin at later times, as is apparent from Figures 10 and 11.

The results at various ranges after summing the first 10 sets of rays are displayed in Figure 12 along with the comparisons with numerical methods. It is relatively expensive to compute the numerical results at large times which is the reason for the truncations [see Boore et al. (1971) and Hong and Kosloff (1978)]. Considering the simplicity of the glorified optics method it is rather surprising that the agreement 
is so good. In general, the beginning portion of each record appears very accurate which corresponds to rays traveling nearly vertical. At later times, the GO results appear less accurate which corresponds to rays traveling more nearly horizontal. This probably means that the curvature of the boundary becomes more important and higher order reflection coefficients may be necessary. Rays that travel from one wall of the basin to the other side without hitting the surface were also considered but found to be weak contributors.

The results with a shorter period time function are given in Figure 13, where some of the amplified arrivals are more apparent such as the first multiple at $x=0$. It is
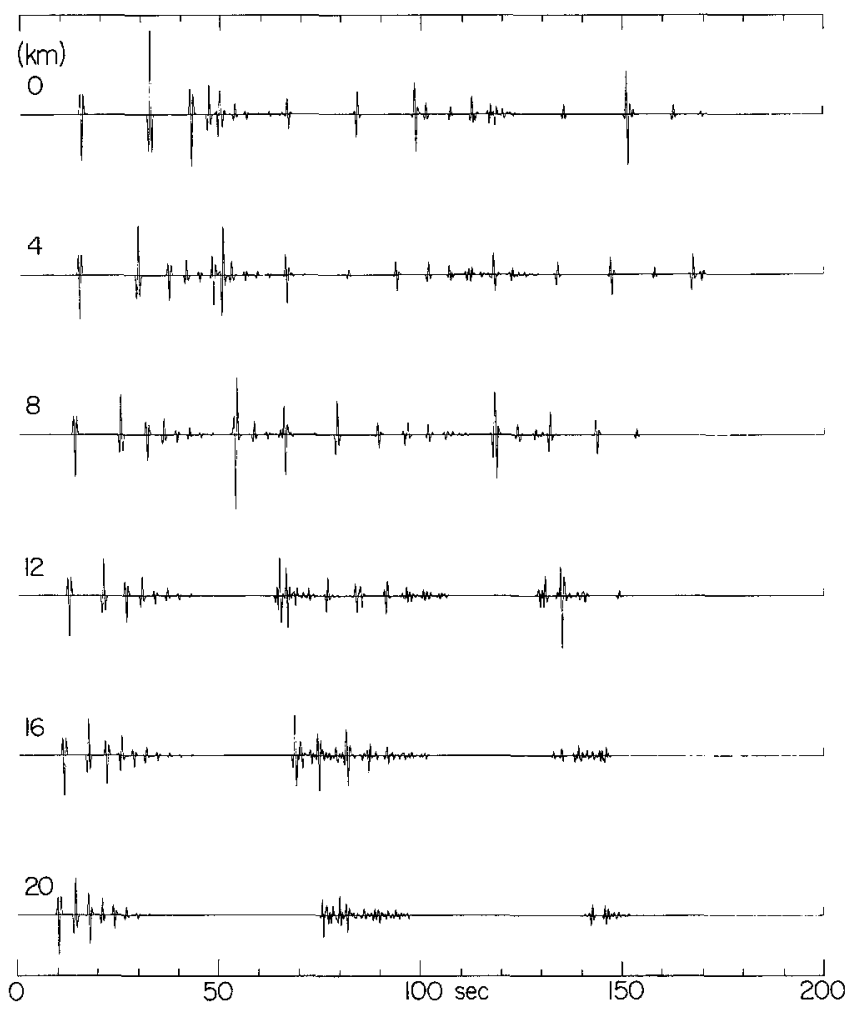

FIG. 13. Diagram showing the $S H$ displacement caused by vertically impinging plane wave with short pulse. The source time function is the Ricker's wavelet [see formula (26)], with $T_{p}=1 \mathrm{sec}, t_{s}=1 \mathrm{sec}$.

relatively important for these large signals to perform the limiting process carefully. That is, we examine the ratio of areas for a narrow beam of area $A_{0}$ and test for stability by considering a series of smaller and smaller values. This process is simple if one requires the boundaries to be smooth and one avoids examining the motion on a caustic. We have not been particularly concerned with the motion at or near caustics in this study since we are primarily interested in developing a methodology for studying seismograms to infer the broad features of structure. On the other hand, the fine geometric detail needed to form caustics in regions of earthquake hazards abound and the role of such focusing could be highly significant.

\section{Discussion}

In general, curved boundaries introduce a number of interesting effects, namely frequency dependent reflection coefficients and geometrical focusing with the latter 
being the dominant feature in the basin study. The reason for this result is chiefly caused by the assumption of plane waves, constant $p$, where we essentially assume infinite travel time compared to the source duration. This assumption would appear to be appropriate for many seismological problems; some examples are the development of body wave codas and wave-form complexity. The interpretation of recordings in terms of synthetics for these applications has been conducted almost exclusively on the simplifying assumption of flat layers. Given the experience developed in the basin study we feel it would be useful to briefly review these subjects.

Most observations of nuclear explosions made at distances between 30 and $90^{\circ}$ are quite simple, especially if the station is located on bedrock as noted by many

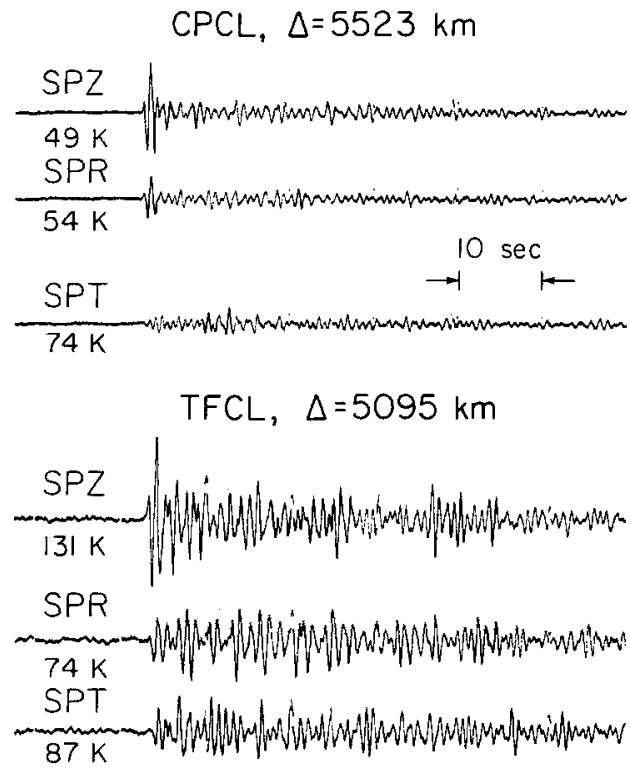

FIG. 14. A comparison of the three components of observed motion of the LONGSHOT nuclear explosion at two sites in Southern California showing the $P$-wave complexity of TFCL (Taft) relative to CPCL (Campo).

authors (see for example Thirlaway, 1966). However, many records show a large complicated coda which has drawn much attention because of the use of $P$-wave complexity as a discriminant between earthquakes and explosions. An example of a simple and a rather complicated $P$ wave is given in Figure 14. Possible explanations have been put forth by numerous authors. Douglas et al. (1971) suggest that the direct $P$ has been attenuated by passing through a lower $Q$ zone relative to the later arrivals. Woodhouse (1973) suggests that strong later arrivals are diffracted arrivals from the upper mantle transition zones. More recent studies (Simpson and Cleary, 1977) suggest that $P$ signal complexity is caused by random scattering along the entire path. It would appear to us that the type of scattering discussed in this paper would be a good explanation of the phenomena in that the scattering could come from shallow structure near the source (Figure 13 in reverse, using the reciprocity principle) and/or the receiver structure. For the example given in Figure 14, we would prefer the latter interpretation since these two stations are at nearly the same ray parameter. The station CPCL is sitting on bedrock whereas TFCL is near the 
edge of the Great Valley. Our proposed technique appears ideally suited to study such problems.

Another application is with wave-form complexity observed in refraction profiling, especially oceanic exploration with OBS receivers. It is common practice to drop OBS's in small basins of sediments to ensure proper coupling to the bottom. However, in many such situations the OBS records are much more complicated than hydrophone recording near the surface of the ocean (see Lewis and McClair, 1977). In this case, converted shear waves could easily become partially trapped and complicate the motion. To test this hypothesis would require a more complete data set. However, in general, the whole question about the intensity and wave-form complexity in the presence of uneven bottom sedimentary cover could be handled with this technique.

Still another interesting application of this technique is with respect to site amplification effects observed in earthquake studies. There is abundant evidence of accelerations in excess of gravity occurring during earthquakes (see for example Morrill, 1972). Numerous authors using numerical codes suggest that 25 per cent of such high values can be attributed to topography effects, Boore (1973), Trifunac (1973), or Bouchon (1973). However, it would appear to be quite easy to generate a factor of two by subsurface focusing at high frequencies of the type discussed in the previous section. But since the observations of large earthquakes contain information from $20 \mathrm{~Hz}$ to static offsets, we must be careful to understand the effects of lateral structure at all wavelengths. Thus, we have started work on including the effects of boundary curvature on curved wave fronts where the duration of signal need not be short compared to the travel time.

In summary, we have presented a practical method of generating synthetic seismograms for models containing nonparallel boundaries. The method consists of summing generalized rays describing the various possible paths based on the ray construction for dipping structure discussed earlier by Hong and Helmberger (1977). The response of each ray is determined by its numerical behavior near its particular arrival time. A comparison between synthetics generated by this new method with those obtained by finite element for a basin structure are presented and a number of possible applications are discussed.

\section{ACKNOWLEDGMENTS}

This work was supported by ONR Contract N00014-76-C-1070 and by the National Science Foundation under Grant ENV76-10506.

\section{REFERENCES}

Aki, K. and K. L. Larner (1970). Surface motion of a layered medium having an irregular interface due to incident plane SH wave, J. Geophys. Res. 75, 933-954.

Boore, D. (1973). The effect of simple topography on seismic waves: implications for the accelerations recorded at Pacoima Dam, San Fernando Valley, California, Bull. Seism. Soc. Am. 63, 1603-1609.

Boore, D. M., K. L. Larner, and K. Aki (1971). Comparison of two independent methods for the solution of wave-scattering problems: Response of a sedimentary basin to vertically incident SH waves, $J$. Geophys. Res. 76, 558-569.

Bouchon, M. (1973). Effect of topography on surface motion, Bull. Seism. Soc. Am. 63, 615-632.

Burdick, L. J. and G. R. Mellman (1976). Inversion of the body waves from the Borrego Mountain Earthquake to the source mechanism, Bull. Seism. Soc. Am. 66, 1485-1499.

Douglas, A., P. D. Marshall, and D. Corbishley (1971). Absorption and the complexity of P signals, Nature Phys. Sci. 232, 50-51.

Hill, D. P. (1974). Phase shift and pulse distortion in body waves due to internal caustics, Bull. Seism. Soc. Am. 64, 1733-1742. 
Helmberger, D. V. (1968). The crust-mantle transition in the Bering Sea, Bull. Seism. Soc. Am. 58, 179-214.

Hong, T. L. and D. V. Helmberger (1977). Generalized ray theory for dipping structure, Bull. Seism. Soc. Am. 67, 995-1008.

Hong, T. L. and D. Kosloff (1978). An application of the finite element method in wave propagation problems involving irregular structures (submitted for publication).

Larner, K. L. (1970). Near-receiver scattering of teleseismic body waves in layered crust-mantle models having irregular interfaces, Thesis, Massachusetts Institute of Technology, Cambridge.

Lewis, B. T. R. and J. McClain (1977). Converted shear waves as seen by ocean bottom seismometers and surface buoys, Bull. Seism. Soc. Am. 67, 1291-1302.

Morrill, B. J. (1972). The San Fernando, California, Earthquake of February 9, 1971, U.S. Geol. Surv. Profess. Paper 733 22, 60-65.

Simpson, D. W. and J. R. Cleary (1977). P-signal complexity and upper mantle structure, Geophys. J. 49, $747-756$.

Thirlaway, H. I. S. (1966). Interpreting array records: Explosion and earthquake $P$ wave trains which have traversed the deep mantle, Proc. Roy. Soc. London 290, 385-395.

Trifunac, M. D. (1973). Scattering of plane SH waves by a semi-cylindrical canyon, Earthquake Eng. Structural Dyn. 1, 267-281.

Woodhouse, J. H. (1973). Diffraction by anomalous regions in the Earth's mantle, Geophys. J. 32, 295-324.

\author{
Division of Geological and Planetary Sciences \\ California Institute of Technology \\ Pasadena, California 91125 \\ Contribution No. 3038
}

Manuscript received February 10, 1978

\title{
APPENDIX
}

In order to help understanding, the derivation of equation (10) is as follows

$$
\begin{gathered}
t=\sum_{m=1}^{3}\left(p_{m} d_{m}+\eta_{m} h_{m}\right) \\
\frac{d^{2} t}{d p_{1}^{2}}=\frac{d}{d p_{1}}\left(\frac{d t}{d p_{1}}\right)=\frac{d}{d p_{1}}\left[\sum_{m=1}^{3}\left(d_{m} \cdot \frac{d p_{m}}{d p_{1}}-h_{m} \cdot \frac{p_{m}}{\eta_{m}} \cdot \frac{d p_{m}}{d p_{1}}\right)\right] \\
=\sum_{m=1}^{3}\left[\left(d_{m}-h_{m} \cdot \frac{p_{m}}{\eta_{m}}\right) \frac{d^{2} p_{m}}{d p_{1}{ }^{2}}-\frac{\left(\eta_{m}{ }^{2}+p_{m}{ }^{2}\right) h_{m}}{\eta_{m}{ }^{2}} \cdot\left(\frac{d p_{m}}{d p_{1}}\right)^{2}\right] \\
\frac{d p_{m}}{d p_{1}}=\frac{d p_{m}}{d p_{m}^{\prime}} \cdot \frac{d p_{m}^{\prime}}{d p_{m-1}} \cdot \frac{d p_{m-1}}{d p_{m-1}^{\prime}} \ldots \frac{d p_{2}}{d p_{2}^{\prime}} \cdot \frac{d p_{2}^{\prime}}{d p_{1}} \\
=\frac{d p_{m}}{d p_{m}^{\prime}} \cdot \frac{d p_{m-1}}{d p_{m-1}^{\prime}} \ldots \frac{d p_{2}}{d p_{2}^{\prime}}
\end{gathered}
$$

where $p_{m}$ and $p_{m}^{\prime}$ are the ray parameters of the $m$-th ray segment, associated respectively with the local coordinate systems suitable to describe the local boundary conditions at its two ends. Note that $p_{m-1}=p_{m}^{\prime}$ by Snell's law. 
At $t=t_{0}$

$$
\begin{gathered}
p_{m}=\frac{\sin \theta_{m}}{v_{m}}, p_{m}^{\prime}=\frac{\sin \theta_{m}^{\prime}}{v_{m}} \\
\eta_{m}=\frac{\cos \theta_{m}}{v_{m}}, \eta_{m}^{\prime}=\frac{\cos \theta_{m}^{\prime}}{v_{m}}
\end{gathered}
$$

where $v_{m}$ is the wave velocity of the medium.

Also,

$$
d_{m}-h_{m} \cdot \frac{p_{m}}{\eta_{m}}=0
$$

Therefore

$$
\begin{aligned}
\left(\frac{d^{2} t}{d p_{1}^{2}}\right)_{t=t_{0}}= & \sum_{m=1}^{3}\left[\frac{-v_{m} h_{m}}{\cos ^{3} \theta_{m}} \cdot\left(\frac{\cos \theta_{m}}{\cos \theta_{m}^{\prime}} \cdot \frac{\cos \theta_{m-1}}{\cos \theta_{m-1}^{\prime}} \cdots \frac{\cos \theta_{2}}{\cos \theta_{2}^{\prime}}\right)^{2}\right] \\
= & \sum_{m=1}^{3}\left[\frac{-v_{m} h_{m}}{\cos { }^{3} \theta_{m}} \cdot \frac{\cos ^{2} \theta_{m}}{\cos ^{2} \theta_{1}} \cdot\left(\frac{\cos \theta_{m-1}}{\cos \theta_{m}^{\prime}} \cdot \frac{\cos \theta_{m-2}}{\cos \theta_{m-1}^{\prime}} \cdots \frac{\cos \theta_{1}}{\cos \theta_{2}^{\prime}}\right)^{2}\right] \\
= & \sum_{m=1}^{3}\left[\frac{-v_{m} h_{m}}{\cos ^{3} \theta_{m}} \cdot \frac{\cos ^{2} \theta_{m}}{\cos ^{2} \theta_{1}} \cdot\left(\frac{v_{m-1} d \theta_{m}^{\prime}}{v_{m} d \theta_{m-1}} \cdot \frac{v_{m-2} d \theta_{m-1}^{\prime}}{v_{m-1} d \theta_{m-2}} \cdots \frac{v_{1} d \theta_{2}^{\prime}}{v_{2} d \theta_{1}}\right)\right. \\
& \left.\cdot\left(\frac{\cos \theta_{m-1}}{\cos \theta_{m}^{\prime}} \frac{\cos \theta_{m-2}}{\cos \theta_{m-1}^{\prime}} \cdots \frac{\cos \theta_{1}}{\cos \theta_{2}^{\prime}}\right)\right] \\
= & \sum_{m=1}^{3}\left[\frac{-R_{m} \cdot v_{1}}{\cos { }^{2} \theta_{1}} \cdot \frac{d \theta_{m}}{d \theta_{1}} \cdot\left(\frac{\cos \theta_{m-1}}{\cos \theta_{m}^{\prime}} \cdot \frac{\cos \theta_{m-2}}{\cos \theta_{m-1}^{\prime}} \cdots \frac{\cos \theta_{1}}{\cos \theta_{2}}\right)\right] .
\end{aligned}
$$

We note that $d \theta_{m}=d \theta_{m}^{\prime}$ since this quantity is invariant with respect to the rotation of coordinate system.

Therefore

$$
\left[\eta_{1}\left|\frac{d^{2} t}{d p_{1}^{2}}\right|^{\frac{1}{2}}\right]_{t=t_{0}}=\frac{1}{\sqrt{v_{1} d \theta_{1}}}\left[\sum_{m=1}^{3} R_{m} d \theta_{m} \cdot\left(\frac{\cos \theta_{m-1}}{\cos \theta_{m}^{\prime}} \cdot \frac{\cos \theta_{m-2}}{\cos \theta_{m-1}^{\prime}} \cdots \frac{\cos \theta_{1}}{\cos \theta_{2}^{\prime}}\right)\right]^{\frac{1}{2}}
$$

